\title{
MicroRNA-140-5p and SMURF1 regulate pulmonary arterial hypertension
}

\author{
Alexander M.K. Rothman, ${ }^{1,2}$ Nadine D. Arnold, ${ }^{1}$ Josephine A. Pickworth, ${ }^{1}$ James Iremonger, ${ }^{1}$ Loredana Ciuclan, ${ }^{3}$ \\ Robert M.H. Allen, ${ }^{3}$ Sabine Guth-Gundel, ${ }^{4}$ Mark Southwood, ${ }^{5}$ Nicholas W. Morrell, ${ }^{5}$ Matthew Thomas, ${ }^{3}$ \\ Sheila E. Francis, ${ }^{1}$ David J. Rowlands, ${ }^{6}$ and Allan Lawrie ${ }^{1}$ \\ 'Department of Infection, Immunity and Cardiovascular Disease, University of Sheffield, Sheffield, United Kingdom. ${ }^{2}$ Sheffield Teaching Hospitals NHS Trust, Sheffield, United Kingdom. \\ ${ }^{3}$ Novartis Institutes for Biomedical Research, Horsham, United Kingdom. ${ }^{4}$ Novartis Institutes for Biomedical Research, Basel, Switzerland. ${ }^{5}$ Department of Medicine, \\ University of Cambridge School of Clinical Medicine, Addenbrooke's and Papworth Hospitals, Cambridge, United Kingdom. ${ }^{6}$ Novartis Institutes for Biomedical Research, Cambridge, Massachusetts, USA.
}

\begin{abstract}
Loss of the growth-suppressive effects of bone morphogenetic protein (BMP) signaling has been demonstrated to promote pulmonary arterial endothelial cell dysfunction and induce pulmonary arterial smooth muscle cell (PASMC) proliferation, leading to the development of pulmonary arterial hypertension (PAH). MicroRNAs (miRs) mediate higher order regulation of cellular function through coordinated modulation of mRNA targets; however, miR expression is altered by disease development and drug therapy. Here, we examined treatment-naive patients and experimental models of PAH and identified a reduction in the levels of miR-140-5p. Inhibition of miR-140-5p promoted PASMC proliferation and migration in vitro. In rat models of PAH, nebulized delivery of miR-140-5p mimic prevented the development of PAH and attenuated the progression of established PAH. Network and pathway analysis identified SMAD-specific E3 ubiquitin protein ligase 1 (SMURF1) as a key miR-140-5p target and regulator of BMP signaling. Evaluation of human tissue revealed that SMURF1 is increased in patients with PAH. miR-140-5p mimic or SMURF1 knockdown in PASMCs altered BMP signaling, further supporting these factors as regulators of BMP signaling. Finally, Smurf1 deletion protected mice from PAH, demonstrating a critical role in disease development. Together, these studies identify both miR-140-5p and SMURF1 as key regulators of disease pathology and as potential therapeutic targets for the treatment of PAH.
\end{abstract}

\section{Introduction}

Pulmonary arterial hypertension $(\mathrm{PAH})$ is a devastating disease characterized by progressive remodeling of the pulmonary vasculature. Genetic predisposition and/or environmental insult result in pulmonary artery endothelial cell (PAEC) apoptosis, pulmonary arterial smooth muscle cell (PASMC) proliferation, occlusive pulmonary vascular remodeling (1-3), increased pulmonary vascular resistance, and right heart failure (4). Current treatments are limited to pharmacological vasodilatation via the prostacyclin, endothelin, or nitric oxide pathways; however, proliferative remodeling persists and many patients require lung transplantation $(5,6)$. New therapeutic approaches are needed to inhibit or reverse vascular remodeling.

Heterozygous mutations in the bone morphogenetic protein receptor 2 (BMPR2) gene are present in approximately $80 \%$ of heritable PAH and in an estimated $20 \%$ of patients with idiopathic $\mathrm{PAH}(7,8)$. In the absence of a recognized mutation, reduced pulmonary vascular expression of BMPR2 has been identified in patients with both idiopathic and associated forms of $\mathrm{PAH}$ (APAH) (9), although the regulatory mechanism of this reduction remains unclear. BMPR2 expression is also reduced in multiple rodent models of PAH (10-13), providing further evidence of its importance in disease pathogenesis. At a cellular level,

Conflict of interest: The authors have declared that no conflict of interest exists. Submitted: June 23, 2015; Accepted: March 31, 2016.

Reference information: J Clin Invest. 2016;126(7):2495-2508. doi:10.1172/JCI83361. suppression of BMP signaling leads to reduced endothelial barrier function $(14,15)$ and increased apoptosis of PAECs $(15,16)$ and drives proliferation $(17,18)$ and apoptosis resistance $(19)$ of PASMCs. Endothelial-specific KO (20) and smooth muscle cell (SMC) expression of a dominant negative BMPR2 (21) both result in the development of PAH in mice. The restoration of endothelial BMPR2 signaling by overexpression (22), intravenous BMPR2 gene therapy $(23,24)$ or exogenous administration of elafin $(25)$ reverses experimental PAH. As such, the BMPR2 signaling pathway represents an attractive target for pharmacological intervention in PAH. The specific BMPR2 agonist BMP9 (15) and clinically approved drugs that nonspecifically enhance BMP signaling, such as FK506 (26), chloroquine (27), and paclitaxel (28), have shown beneficial effects in experimental PAH. These studies and the positive clinical effect of FK506 demonstrated during compassionate use (28) highlight the potential benefits of enhanced BMP signaling as a treatment for PAH.

MicroRNAs (miRs) are short noncoding RNAs that mediate posttranscriptional regulation through interactions of their seed region with complementary sequences in the $3^{\prime}$ UTR of target mRNA (29). Through the simultaneous repression of multiple targets, miRs mediate higher order regulation of cellular function. Both increased and decreased miR expression have been implicated in the pathology of PAH, and the therapeutic utility of modulating miR expression has been demonstrated in experimental PAH. The investigation of miR dysregulated in human disease and the cellular mechanisms through which dysregulated 
A

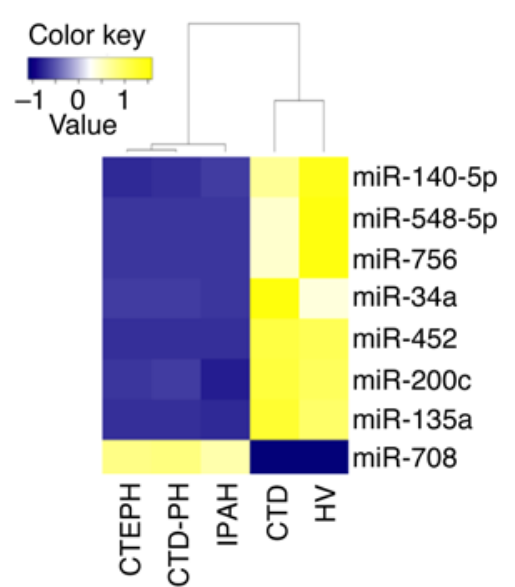

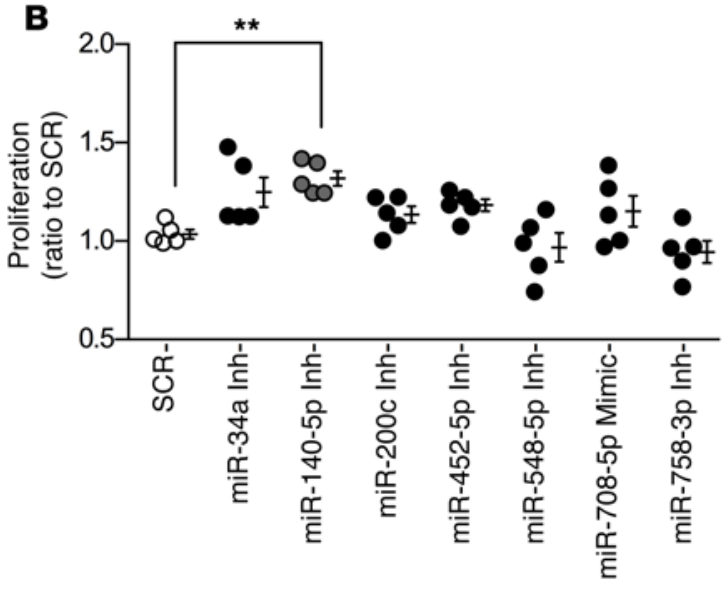
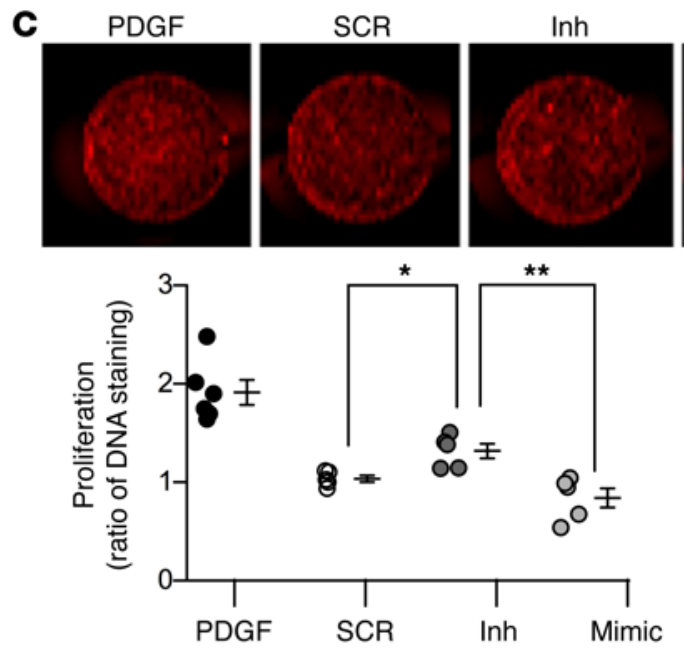

E

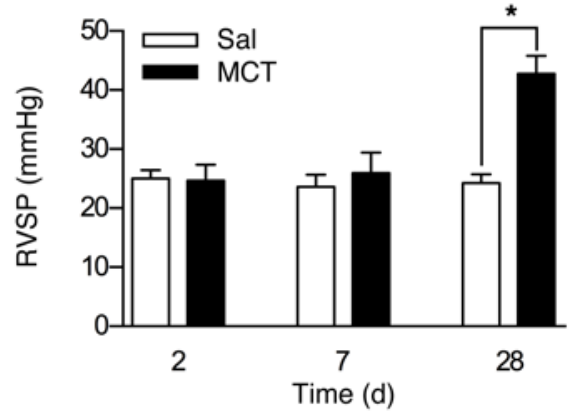

G

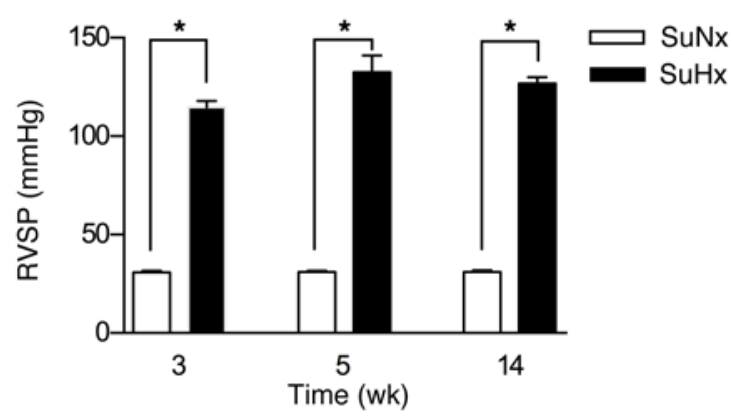

Mimic
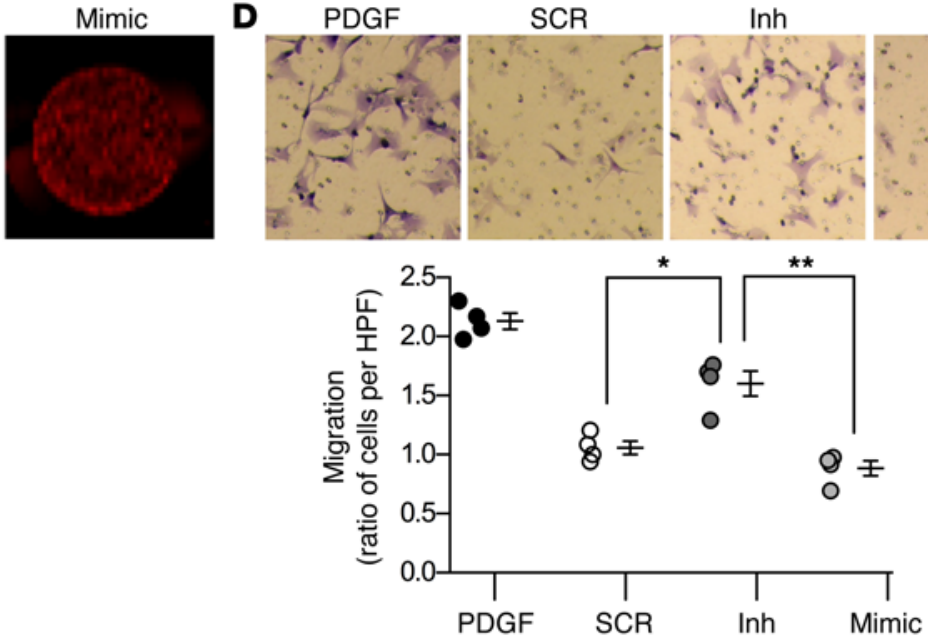

$\mathbf{F}$

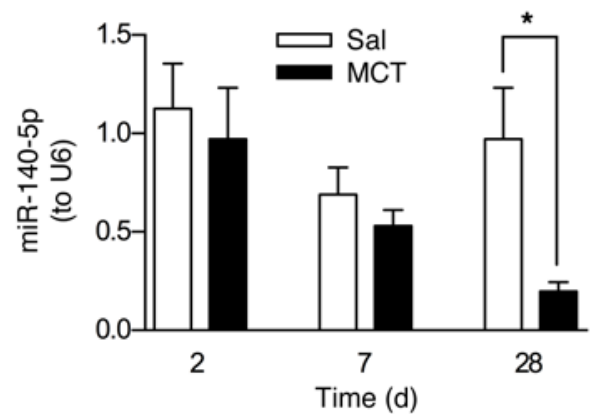

H

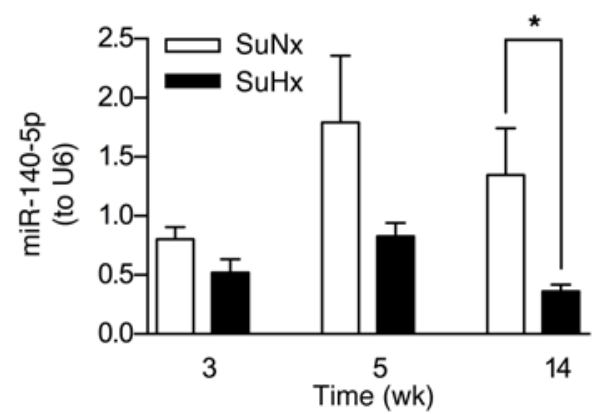


Figure 1. miR-140-5p is reduced in patients with $\mathrm{PH}$ and animal models of PAH and modulates PASMC phenotype. (A) Heat map representing differentially expressed whole blood miR in treatment-naive patients with $\mathrm{PH}$ and control groups (fold change $>2$, comparison between patients with $\mathrm{PH}$ and control groups, $n=4$ per group). (B) Effect of whole blood miR on PASMC proliferation in vitro. PASMCs were transfected with miR mimic, inhibitor (Inh), or scramble control (SCR) and proliferation assessed at 72 hours. Inhibition of miR-135a led to cell death, and inhibition of miR140-5p led to increased proliferation $\left(n=5,{ }^{*} P<0.05\right.$, 1-way ANOVA with Dunnett's post-test correction, mean \pm SEM). (C and D) PASMC proliferation (C) and migration (D) following miR-140-5p mimic and inhibitor transfection at 72 and 6 hours, respectively. miR-140-5p inhibitor led to increased proliferation and migration in comparison with both SCR and miR-140-5p mimic (C: $n=5, \mathbf{D}: n=4, \mathbf{C}$ and $\mathbf{D}:{ }^{*} P<0.05,{ }^{*} P<0.01,1$-way ANOVA with Tukey's post-test correction, mean \pm SEM, photomicrographs representative of $n=5$ (C), and $n=4$ (D). Original magnification, $\times 10$ (C); $\times 20$ (D). (E and F) miR-140-5p is reduced during development of PAH in the MCT rat model. RVSP (E) is increased and miR-140-5p (F) decreased at day 28 following injection of $60 \mathrm{mg} / \mathrm{kg}$ MCT in the Sprague-Dawley rat (E and $\mathbf{F}, n=5-8$ per group, ${ }^{*} P<0.05$, 2-way ANOVA with Tukey's post-test correction, mean $\pm \mathrm{SEM}$ ). ( $(\mathbf{C}$ and $\mathbf{H}$ ) miR-140-5p is reduced during the development of PAH in the SuHx rat model. RVSP is increased and miR140-5p decreased at week 14 following injection with $20 \mathrm{mg} / \mathrm{kg}$ Su5416 and 3 weeks of $10 \% \mathrm{O}_{2}$ in the Wistar-Kyoto rat ( $\mathbf{G}$ and $\mathbf{H}: n=8$ per group, ${ }^{*} P<0.05,2$-way ANOVA with Tukey's post-test correction, mean \pm SEM).

miRs' effects are mediated may identify key regulators of disease pathology and novel therapeutic targets.

The expression of miR in experimental models of PAH is dependent on the time point and model examined (30). Treatment of patients with PAH-specific therapies alters signaling in diseaserelevant pathways (31). To exclude the effect of PAH-specific drug therapy on cell signaling and minimize variation in the clinical disease state, we examined whole blood miR expression in patients at the time of diagnosis with PAH prior to the initiation of drug therapy. We identified downregulation of miR-140-5p, a tumor-suppressor miR that regulates cellular proliferation and migration, reduced levels of which have been reported in patients with hepatocellular carcinoma (32) and lung (33) and breast cancer $(34,35)$. Consistent with our clinical findings, levels of miR-140-5p were reduced at later time points in experimental models of PAH. miR-140-5p inhibitor increased proliferation and migration of PASMCs, suggesting a potential therapeutic role for miR-140-5pmimic and implicating key derepressed targets in disease pathology. Delivery of miR-140-5p mimic prevented the development of experimental PAH and attenuated the progression of established disease. Bioinformatic analysis identified BMP signaling as a pathway altered by miR-140-5p and highlighted a number of targets with known and previously undetermined roles in disease pathology. SMAD-specific E3 ubiquitin protein ligase 1 (SMURF1), an E3 ubiquitin ligase that targets BMPR2 and downstream signaling mediators for proteasomal degradation, was central within the network of pulmonary hypertension-related (PH-related) miR-140-5p targets and had the highest conserved predicted binding site, with miR-140-5p. Expression of SMURF1 was increased in whole blood samples of treatmentnaive patients with PAH and SMURF1 immunoreactivity increased within the remodeled pulmonary vasculature of patients with heritable and APAH. The role of SMURF1 in the development of PAH is unknown, and its binding site with miR-140-5p is unvalidated. We therefore validated the miR-140-5p/SMURF1-binding site in
PASMC and demonstrated augmentation of BMP signaling with miR-140-5p mimic and SMURF1 siRNA. Finally, genetic ablation of Smurf1 resulted in an allele-dependent protection from experimental PAH. These findings demonstrate the feasibility of targeting BMP signaling for the treatment of PAH.

\section{Results}

Expression of miR-140-5p is reduced in patients with $\mathrm{PH}$. To study whole blood expression of $\mathrm{miR}$ in patients with disease prior to the initiation of drug therapy, we performed real-time quantitative PCR (qPCR) array studies (TaqMan Human MicroRNA V2.0 A, Invitrogen) on samples taken at time of diagnosis from 3 groups of patients with $\mathrm{PH}$ (idiopathic PAH [IPAH], connective tissue disease [CTD] with PH [CTD-PH], and chronic thromboembolic $\mathrm{PH}$ [CTEPH]) and matched control samples from healthy volunteers (HV) and patients with CTD with normal pulmonary artery pressures (Supplemental Table 1; supplemental material available online with this article; doi:10.1172/JCI83361DS1). Expression of 7 miRs was decreased (miR-34a, miR-135a, miR-140-5p, miR-200c, miR-452-5p, miR-548-5p, and miR-758-3p), and 1 (miR-708-5p) was increased in patients with PH (Figure 1A). qPCR validation of individual patient samples demonstrated a significant downregulation of miR-140-5p and miR-200c and an upregulation of miR708 (Supplemental Figure 1).

miR-140-5p modulates PASMC phenotype. To investigate the phenotypic effect of altered levels in a disease-relevant cell, human PASMCs were transfected with miR mimic or inhibitor to replicate the conditions identified in whole blood. Transfection of PASMCs with miR-135a inhibitor resulted in cell death. Transfection with miR-140-5p inhibitor led to increased proliferation (Figure 1, B and C) and migration (Figure 1D).

miR-140-5p is reduced in experimental models of PAH. To further investigate the biological relevance of downregulation of miR140-5p, we examined expression in 2 experimental models of $\mathrm{PAH}$. Consistent with previous studies, miR expression varied during disease development in experimental models (30). However, the development of severe disease led to downregulation of miR140-5p at day 28 in whole lung total RNA samples from animals with PAH induced by administration of monocrotaline (MCT) (Figure 1, E and F) and at week 14 in whole blood samples from rats with PAH induced by administration of the vascular endothelial growth factor receptor inhibitor SU5416 and hypoxia (SuHx) (Figure 1, G and $\mathrm{H}$ ). Thus, consistent human disease downregulation of miR140-5p is a consistent finding in established experimental PAH.

miR-140-5p is reduced in patients with idiopathic $P A H$. To investigate the relationship between miR-140-5p and clinical severity of disease, levels were determined in a cohort of 20 patients with IPAH and 16 age-matched controls (HV) (Supplemental Table 2). Expression of miR-140-5p was reduced in patients at the time of diagnostic right heart catheterization (Figure 2A). Although there was overlap between groups, low levels of miR-140-5p identified patients with IPAH (Figure 2B) and, furthermore, identified those with adverse prognosis (Figure 2C). Low levels of miR-140-5p were also associated with indicators of increased disease severity used in clinical practice for risk stratification and treatment optimization (WHO functional class, exercise capacity, right atrial pressure, and cardiac index) (ref. 6 and Figure 2, D-G). 
A

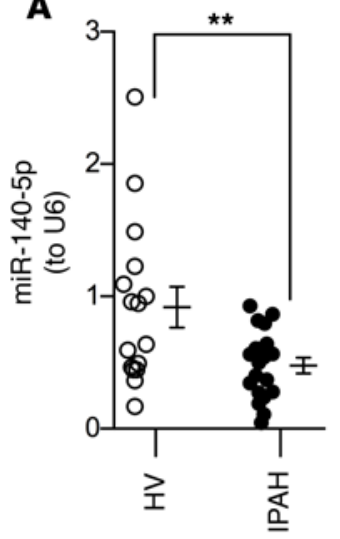

B

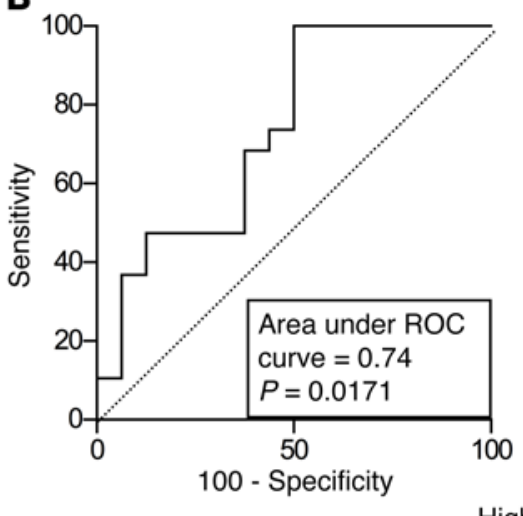

C

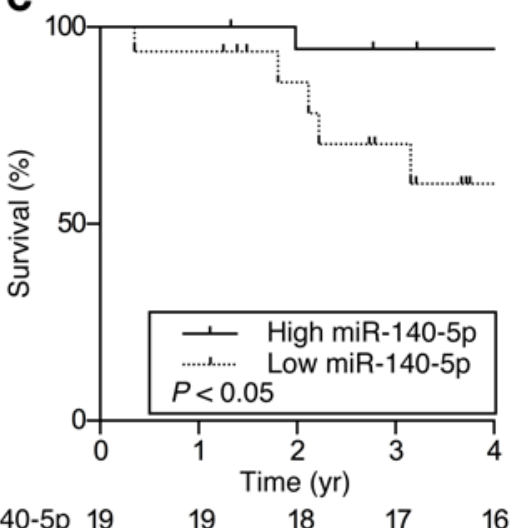

D

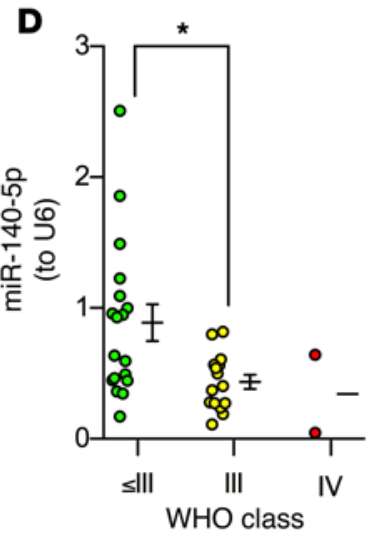

E

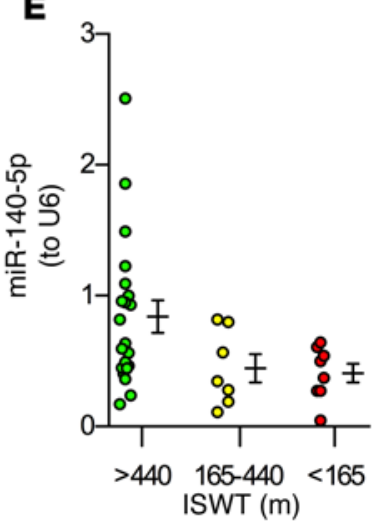

High miR-140-5p 19 Low miR-140-5p 16

$\mathbf{F}$

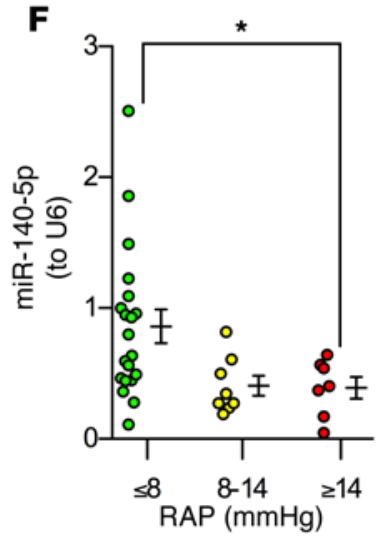

$\begin{array}{llll}15 & 18 & 17 & 16 \\ 15 & 11 & 7 & 2\end{array}$

G

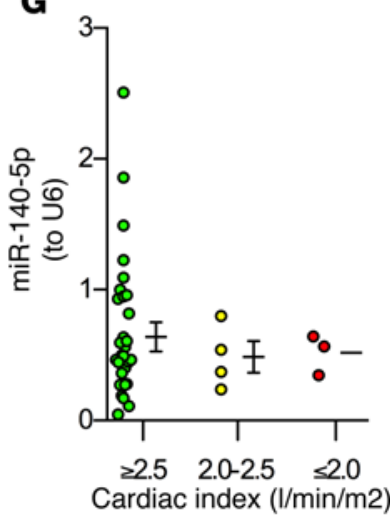

Figure 2. miR-140-5p is reduced in patients with IPAH. (A) Lower whole blood levels of miR-140-5p were observed in patients with IPAH when compared with HV (IPAH, $n=20, H V, n=16,{ }^{* *} P<0.01$, unpaired 2-tailed Student's $t$ test, mean \pm SEM). (B) Receiver operating characteristic (ROC) curve showing sensitivity and specificity of whole blood miR-140-5p levels for discriminating patients with PAH from HV at time of diagnosis (IPAH, $n=20, \mathrm{HV}$, $n=16$, area under ROC curve $=0.74, P=0.0171$ ). (C) Kaplan-Meier analysis of survival, stratified by ROC-derived miR-140-5p cut-off. Survival in patients with miR-140-5p levels above the cut-off is indicated by the solid line and survival in patients with levels below by the dashed line. The table beneath the graph indicates numbers at risk over time in years (IPAH, $n=20, \mathrm{HV}, n=16,{ }^{*} P<0.05$, log-rank [Mantel-Cox] test). (D-G) Reduced levels of miR-140-5p are present in patients with increasing clinical severity of PAH: WHO class (D), incremental shuttle walk test distance (ISWT) (E), right atrial pressure (RAP) (F), and cardiac index (G) (D-G: IPAH, $n=20, \mathrm{HV}, n=16,{ }^{*} P<0.05$, 1-way ANOVA with Dunnett's post-test correction, mean \pm SEM).

Treatment with nebulized miR-140-5p mimic prevents the development of experimental PAH. miR-140-5p expression was decreased with the development of disease in the MCT rat model of PAH (Figure 1, E and F). To explore whether miR-140-5p mimic would prevent the development of disease phenotype, rats were injected with MCT and randomly assigned to blinded treatment groups receiving nebulized delivery of miR-140-5p mimic or scramble control miR at days 0,7 , and 14 (Figure $3 \mathrm{~A}$ and ref. 36). Rats treated with scramble control miR developed PAH as defined by decreased pulmonary artery acceleration time (PAAT) (Figure 3B) and elevated right ventricular systolic pressure (RVSP) (Figure 3C). The miR140-5p mimic-treated group had a longer PAAT (Figure 3B) and reduced RVSP (Figure 3C). There were no significant differences in left ventricular hemodynamics between groups (Supplemental Table 3). Confirming delivery, levels of miR-140-5p were increased in whole lung RNA extract of mimic-treated animals at the time of catheterization. However, levels 1 week after the final nebulized dose were lower than those in control Sprague-Dawley rats (Figure 3D). miR-140-5p mimic-treated rats also showed a reduction in the degree of pulmonary vascular remodeling (Figure $3 \mathrm{E}$ ), as measured by vessel muscularization (Figure $3 \mathrm{~F}$ ), and media to cross-sectional area (Figure 3G), which was associated with fewer proliferating cell nuclear antigen-positive (PCNA-positive) cells within the media of remodeled pulmonary arterioles (Figure $3 \mathrm{E}$ ).

Treatment with nebulized miR-140-5p mimic attenuates established disease in 2 experimental models of PAH. Data presented thus far offer evidence that miR-140-5p modulates PASMC phenotype in vitro and prevents disease development in the MCT rat. We next investigated whether miR-140-5p mimic would alter the progression of established disease in both the MCT and SuHx rat models of PAH.

First, animals were injected with MCT and allowed to develop PAH over 21 days prior to experimentation (Figure 4A). The presence of disease was confirmed by echocardiography (Figure $4 \mathrm{~B})$, and animals were randomly assigned to blinded treatment groups receiving nebulized delivery of miR-140-5p mimic or scramble control miR (Figure 4A). Hemodynamic and pathological parameters in all groups indicated development of PAH. However, the miR-140-5p mimic-treated group had an increased PAAT (Figure 4B) and reduced RVSP (Figure 4C) and right ventricular hypertrophy (RVH) (Figure 4D) compared with scramble control miR-treated animals, indicating therapeutic effect on established MCT-induced PAH. Confirming delivery, levels of 
A
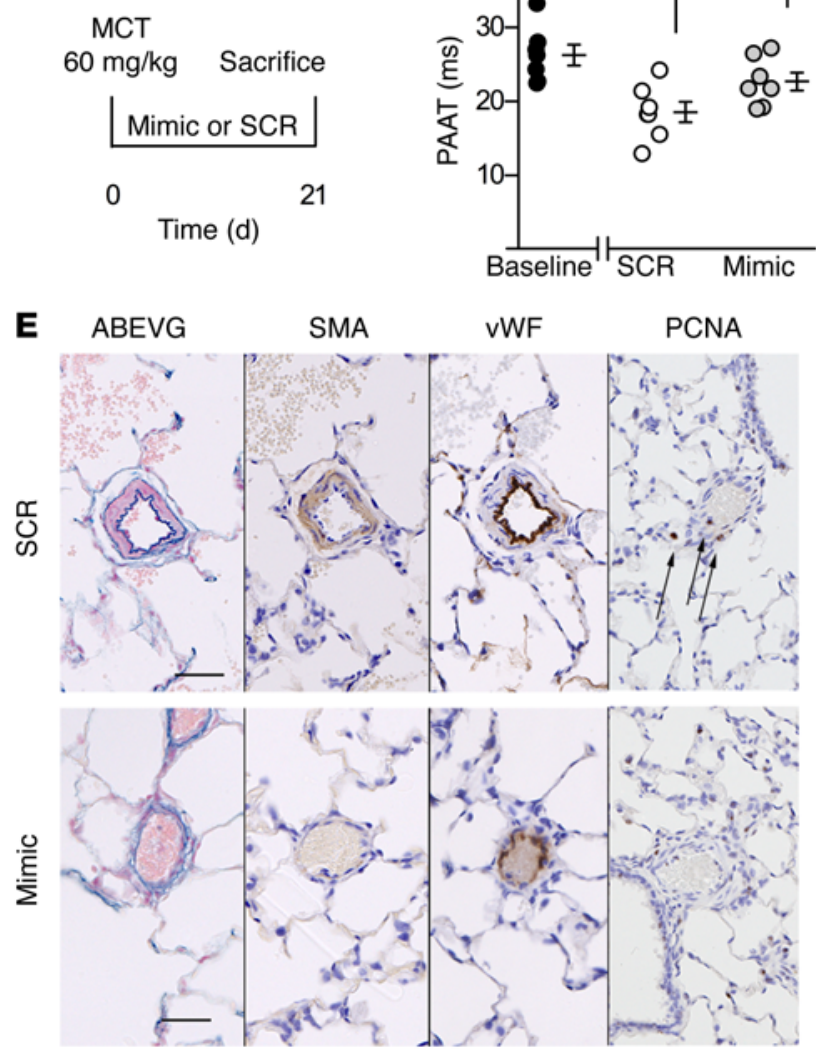

B

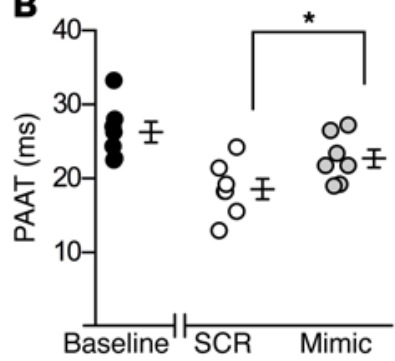

C
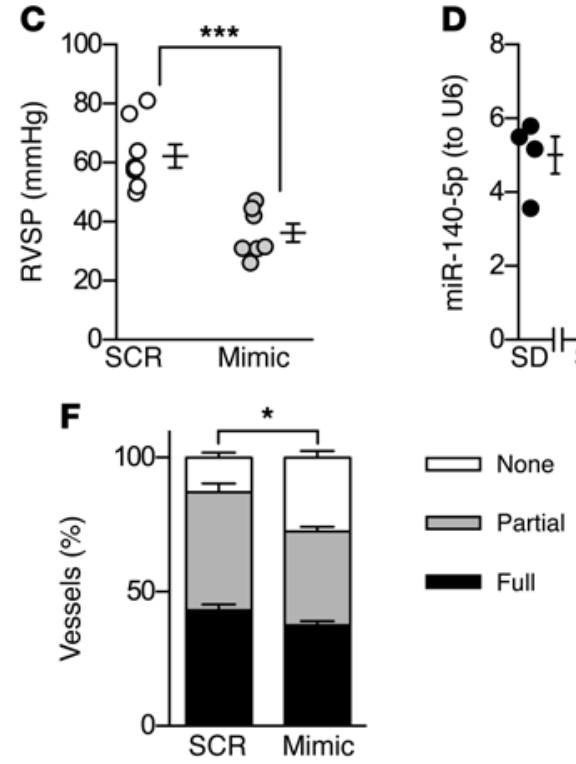

G

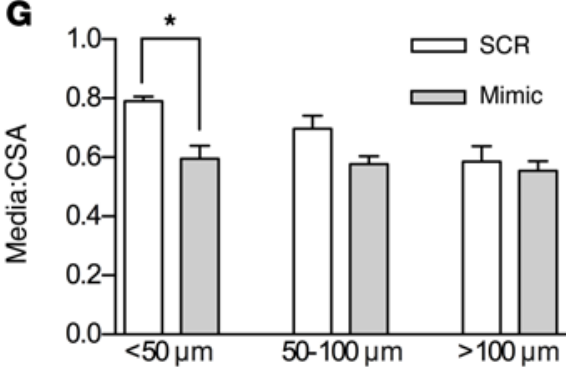

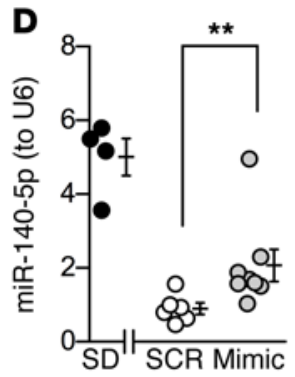

와

Figure 3. miR-140-5p prevents the development of PAH in the MCT rat model. (A) Experimental time line. (B) PAAT at days 0 and 21. (C) RVSP at day 21. (D) qPCR showing whole lung miR-140-5p levels at day 21. (E) Representative photomicrographs of lung sections from SCR and miR-140-5p mimic-treated animals at day 21. Sections stained with Alcian blue EVG, $\alpha$-smooth muscle actin (SMA), vWF, and PCNA (photomicrographs representative of $n=7-8$ per group). Original magnification, $\times 200$. Scale bars: $50 \mu \mathrm{m}$. (F and $\mathbf{G}$ ) Pulmonary vascular remodeling by percentage muscularized vessels (F) and medial wall thickness as a ratio of total vessel size (media/CSA) (C) (B-G: $n=7-8$ per group, ${ }^{*} P<0.05 ;{ }^{* *} P<0.01$; ${ }^{* *} P<0.001$, 2-tailed Mann-Whitney $U$ test, mean \pm SEM).

miR-140-5p were increased in whole lung RNA extract of mimictreated animals at time of catheterization. However, levels 1 week after the final nebulized dose were lower than those in control Sprague-Dawley rats (Figure 4E). No significant changes in left ventricular hemodynamics were observed (Supplemental Table 4). miR-140-5p mimic-treated rats also showed a reduction in the degree of pulmonary vascular remodeling (Figure $4, \mathrm{~F}-\mathrm{H}$ ).

Second, animals were treated with SU5416 and 3 weeks of hypoxic exposure followed by a further 5 weeks in normoxic conditions prior to experimentation (Figure 5A). The presence of disease was confirmed by echocardiography (Figure 5B), and animals randomly assigned to blinded treatment groups received nebulized delivery of miR-140-5p mimic or scramble control miR (Figure 5A). Hemodynamic and pathological parameters in all groups indicated development of PAH. However, animals treated with miR-140-5p mimic had an increased PAAT (Figure 5B) and reduced RVSP (Figure 5C) and RVH (Figure 5D) compared with scramble control miR-treated animals, indicating a therapeutic effect on established SuHx-induced PAH. No significant changes in left ventricular hemodynamics were observed (Supplemental Table 5). Confirming delivery, levels of miR-140-5p were increased in whole lung RNA extract of mimic-treated animals at the time of catheterization (Figure 5E), and Cy3-labeled probe was detected in the pulmo- nary vasculature (Supplemental Figure 2). However, levels 1 week after the final nebulized dose were lower than those in control Wistar Kyoto rats (Figure 5E). As demonstrated in both MCT studies, miR-140-5p mimic-treated rats also showed a reduction in the degree of pulmonary vascular remodeling (Figure $5, \mathrm{~F}-\mathrm{H}$ ).

miR-140-5p regulates key mediators of PAH pathogenesis. Pathway analysis of miR-140-5p targets identified overrepresentation in pathways related to $\mathrm{PAH}$ pathology (Supplemental Table 6). To refine potential target genes downstream of miR-140-5p that may be specifically involved in the pathogenesis of $\mathrm{PAH}$, we identified targets within a PH-related gene network (refs. 37, 38, and Supplemental Table 7). The predicted miR-140-5p network includes key genes regulating multiple points in the TGF- $\beta$ superfamily (Figure 6A, Supplemental Table 8, and Supplemental Figure 3). Regulation of a target mRNA by an miR requires temporal and spatial coexpression. We therefore tested predicted miR-140-5p-target interactions in PASMCs. Transfection with miR-140-5p inhibitor increased, and mimic decreased, mRNA and protein expression of TGF- $\beta$ receptor 1 (ALK5), platelet-derived growth factor receptor $\alpha$ (PDGFRA) and SMURF1, validating bioinformatic predictions in a disease-relevant cell (Figure 6, B and C).

SMURF1 is increased in patients with $P A H$, and levels are regulated by miR-140-5p. Within the miR-140-5p PH-related gene 
A

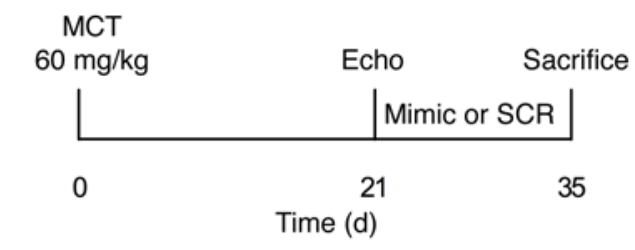

C

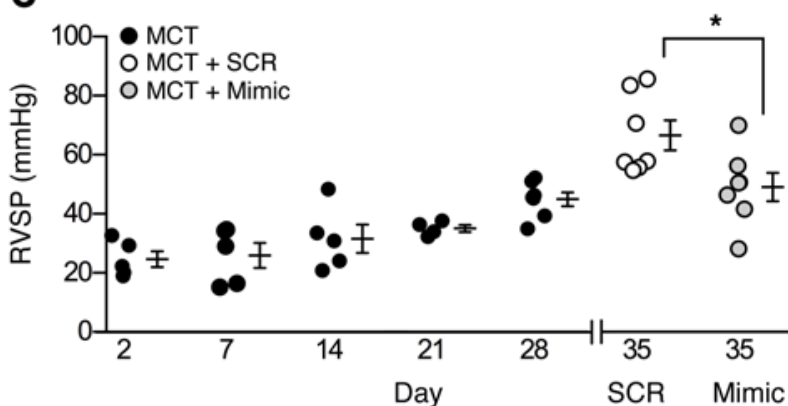

$\mathbf{F}$

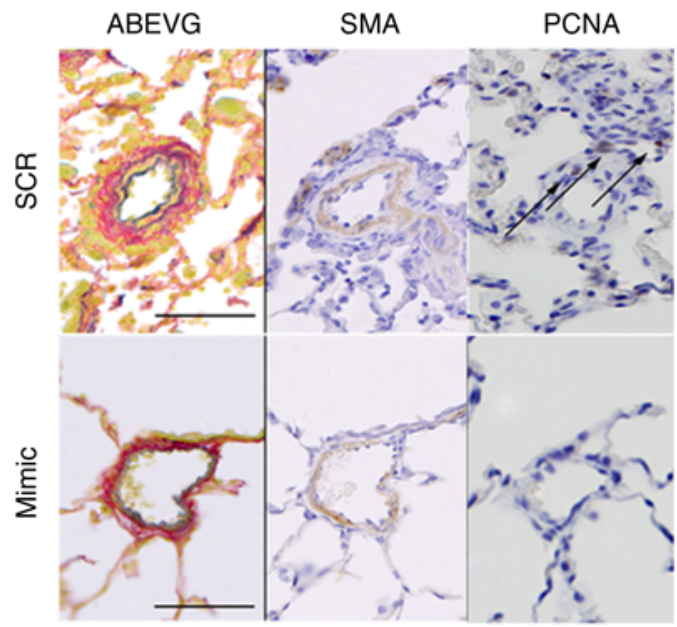

B

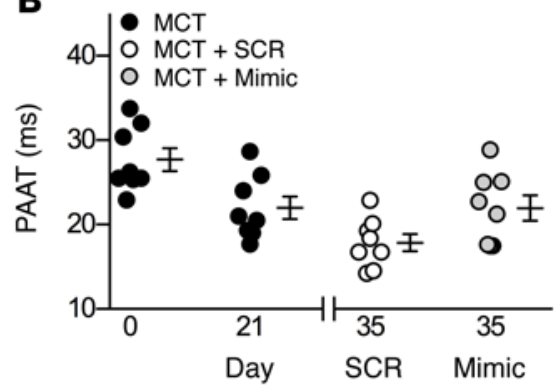

D

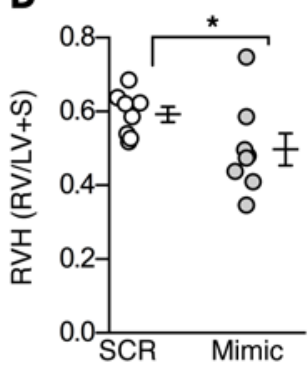

E

G

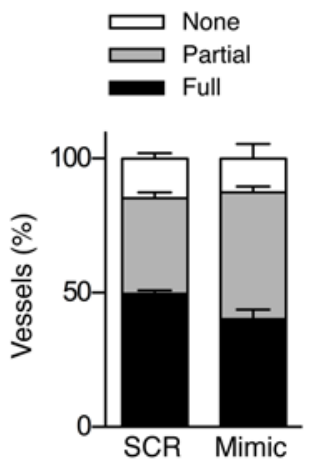

Figure 4. miR-140-5p attenuates experimental PAH in the MCT rat model of PAH. (A) Experimental time line. (B) PAAT at days 0,21 , and 35 . (C) RVSP at day 35. (D) RVH at day 35. (E) qPCR showing whole lung miR-140-5p levels at day 35. SD, Sprague-Dawley; S, SCR; M, mimic. (F) Representative photomicrographs of lung sections from SCR and miR-140-5p mimic-treated animals at day 35. Sections stained with Alcian blue EVG, $\alpha$-SMA, and PCNA (photomicrographs representative of $n=7-8$ per group). Original magnification, $\times 200$. Scale bars: $50 \mu \mathrm{m}$. (G and $\mathbf{H}$ ) Pulmonary vascular remodeling by percentage of muscularized vessels (C) and medial wall thickness as a ratio of total vessel size (media/CSA) (H) (B-H: $n=7-8$ per group, ${ }^{*} P<0.05,2$-tailed Mann-Whitney $U$ test, mean \pm SEM).

network, the most connected protein and the target with the greatest preferentially conserved targeting score was SMURF1 (Figure 6A, Supplemental Tables 9 and 10, and ref. 39). Whole lung SMURF1 protein correlated inversely with miR-140-5p (Figure 6D) in experimental studies, suggesting a regulatory interaction. Further increasing confidence in SMURF1 as an important mediator of disease pathology, SMURF1 mRNA was increased in whole blood from treatment-naive patients at the time of diagnosis with PAH (Figure 6E) and SMURF1 protein immunoreactivity increased within endothelial and SMCs of pulmonary vascular lesions of explanted lungs from patients with BMPR2 mutation-associated heritable PAH and associated PAH (Figure 6F).
miRs mediate posttranscriptional regulation by direct binding of sequences analogous to their seed region in the $3^{\prime}$ UTR of target mRNA (Figure 6G). To demonstrate direct targeting of the SMURF1 3' UTR by miR-140-5p, we transfected PASMCs with a plasmid containing a luciferase-SMURF1 3' UTR construct. Cotransfection with miR-140-5p mimic or inhibitor increased and decreased luciferase activity, respectively (Figure 6, H and I).

miR-140-5p and SMURF1 modulate BMP signaling in PASMCs. The data presented thus far provide evidence that reduced levels of miR-140-5p contribute to the pathogenesis of PAH through derepression of key cellular targets. Target prediction and network analysis identified SMURF1 as a key miR-140-5p target and as a regulator of BMP signaling in PAH. 
A
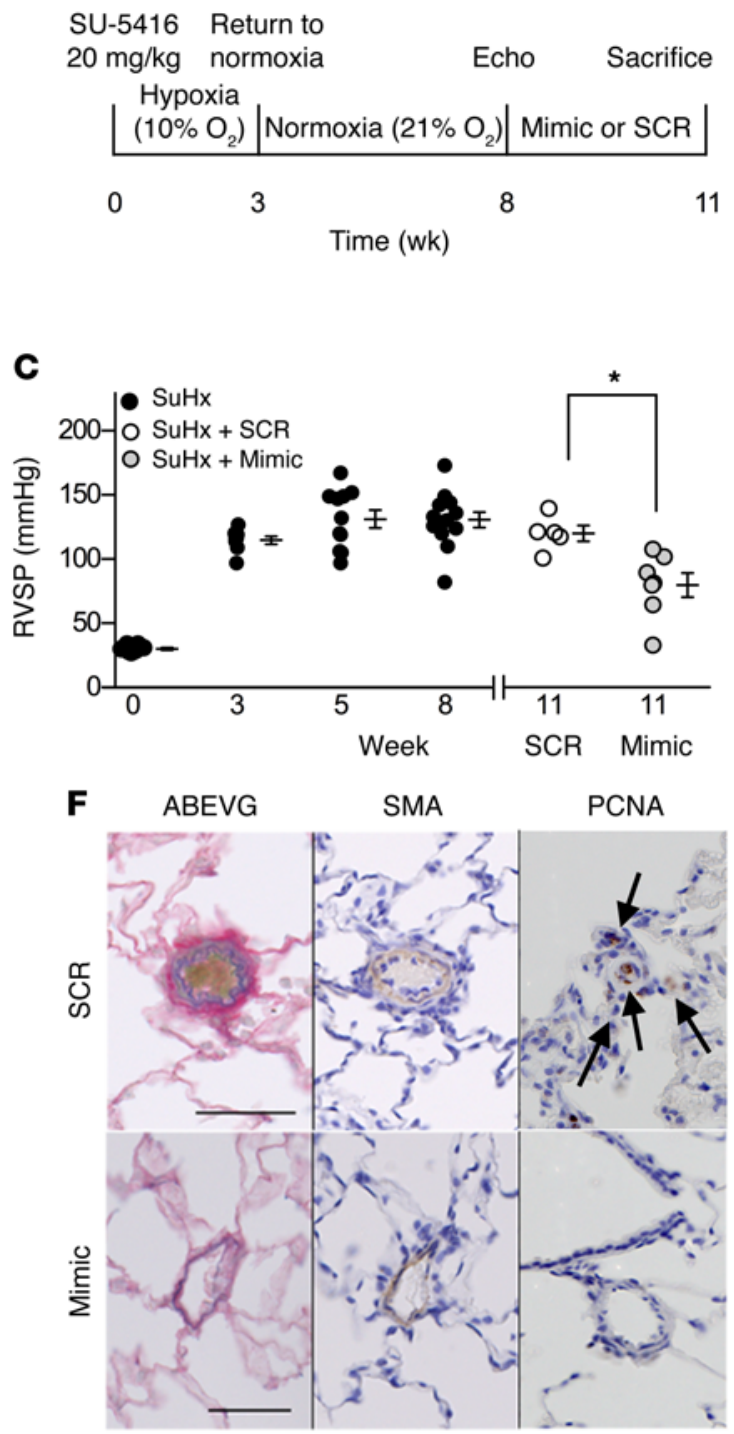
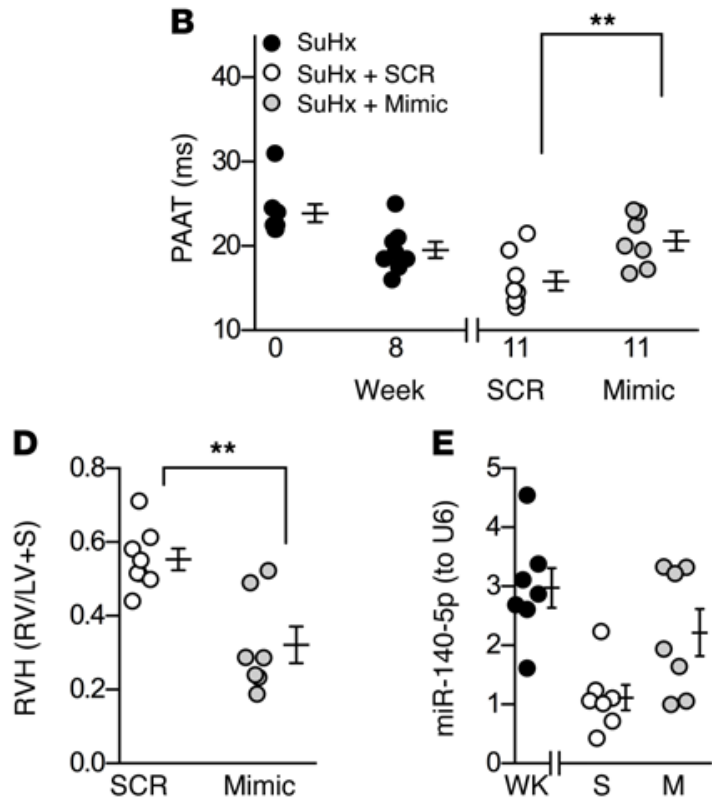

G

H

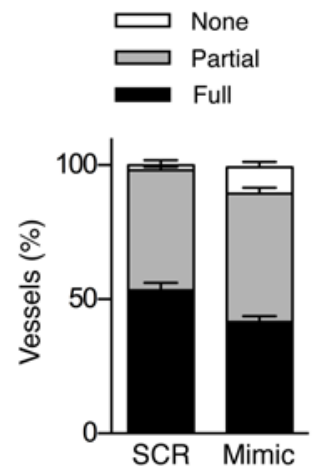

Figure 5. miR-140-5p attenuates experimental PAH in the Sugen hypoxic rat model of PAH. (A) Experimental time line. (B) PAAT at weeks 0, 8, and 11. (C) RVSP at week 11. (D) RVH at 11 weeks. (E) qPCR showing whole lung miR-140-5p levels at 11 weeks. (F) Representative photomicrographs of lung sections from SCR and miR-140-5p mimic-treated animals at 11 weeks. Sections stained with Alcian blue EVG, $\alpha$-SMA, and PCNA (photomicrographs representative of $n=5-7$ per group). Original magnification, $\times 200$. Scale bars: $50 \mu \mathrm{m}$. (G and $\mathbf{H})$ Pulmonary vascular remodeling by percentage of muscularized vessels (G) and medial wall thickness as a ratio of total vessel size (media/CSA) (H). (B-H: $n=5-7$ per group, ${ }^{*} P<0.05,{ }^{*} P<0.01,2$-tailed Mann-Whitney $U$ test, mean \pm SEM).

Reduced BMP signaling is a common pathological finding in $\mathrm{PAH}$, and augmentation of this pathway may provide therapeutic benefit. Our network and pathway analysis (Supplemental Tables 2 and 4 and Supplemental Figure 3) indicated that both miR140-5p and SMURF1 regulate BMP signaling, activity that is indicated by expression of the transcription factor inhibitor of differentiation 1 (ID1) (40). To demonstrate modulation of BMP signaling by SMURF1 and miR-140-5p, PASMCs were transfected with nontargeting siRNA or siRNA to SMURF1 and SCR or miR-140-5p mimic prior to stimulation with BMP4. SMURF1 siRNA and miR140-5p mimic both increased BMP4-mediated signaling, as demonstrated by increased expression of ID1 protein (Figure 7A) and increased activity of an ID1 promoter luciferase construct (BMP response element [BRE]) (Figure 7, B and C).
Genetic ablation of Smurf1 prevents the development of PAH. SMURF1 is increased in patients with PAH, is regulated by miR140-5p, and regulates BMP signaling in PASMCs. To investigate the role of SMURF1 in the development of PAH, we studied the effect of genetic deletion of Smurf1 in C57BL/6 mice exposed to SuHx. Loss of Smurf1 resulted in an allele-dependent protection from the development of PAH as shown by reduced RVSP (Figure 7D), RVH (Figure 7E), and lung remodeling (Figure 7F), demonstrating the critical importance of SMURF1 in PAH pathogenesis.

\section{Discussion}

The present study is the first, to our knowledge, to identify downregulation of miR-140-5p in treatment-naive patients and experimental models of PAH. Treatment with miR-140-5p mimic 

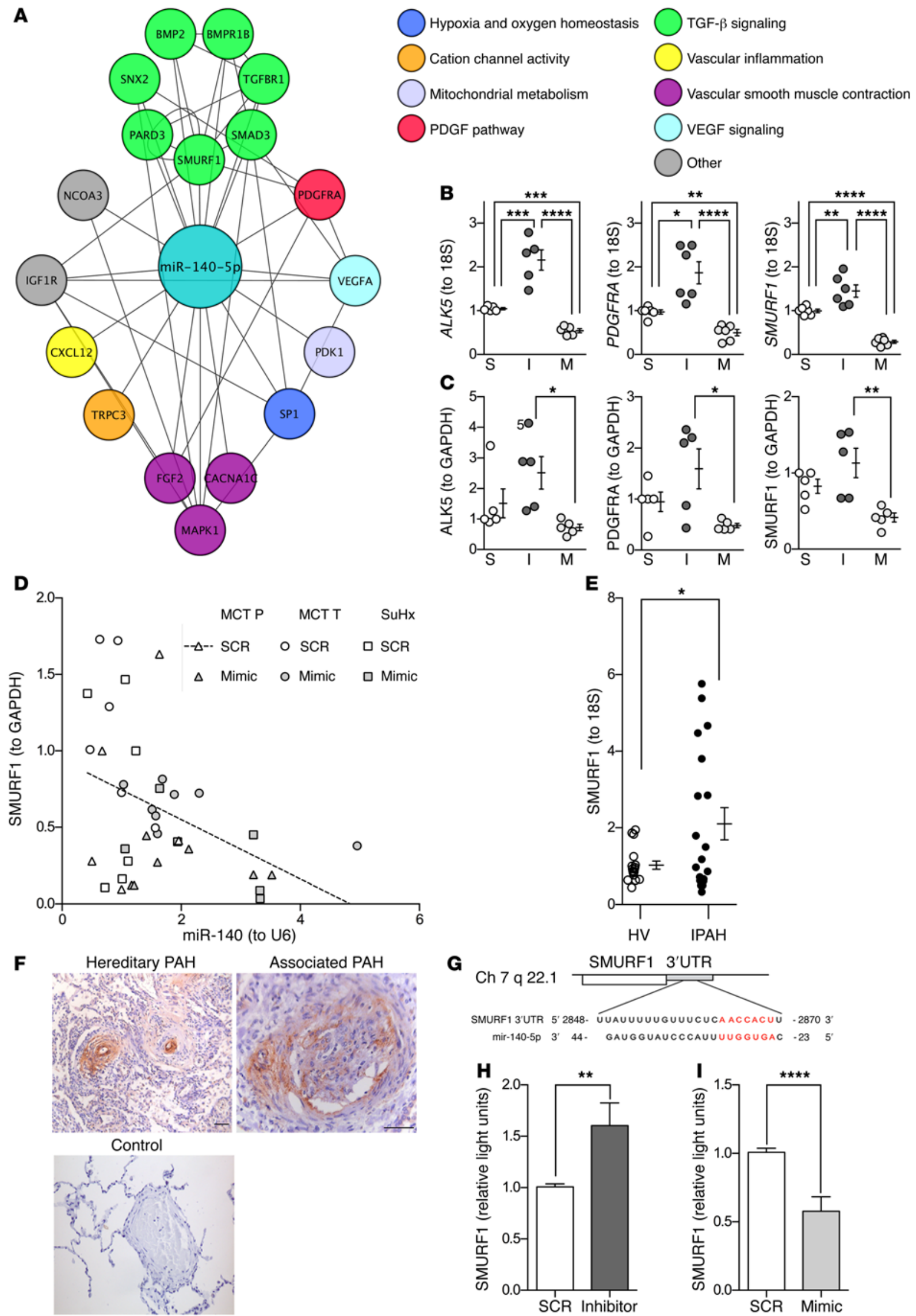

Associated $\mathrm{PAH}$
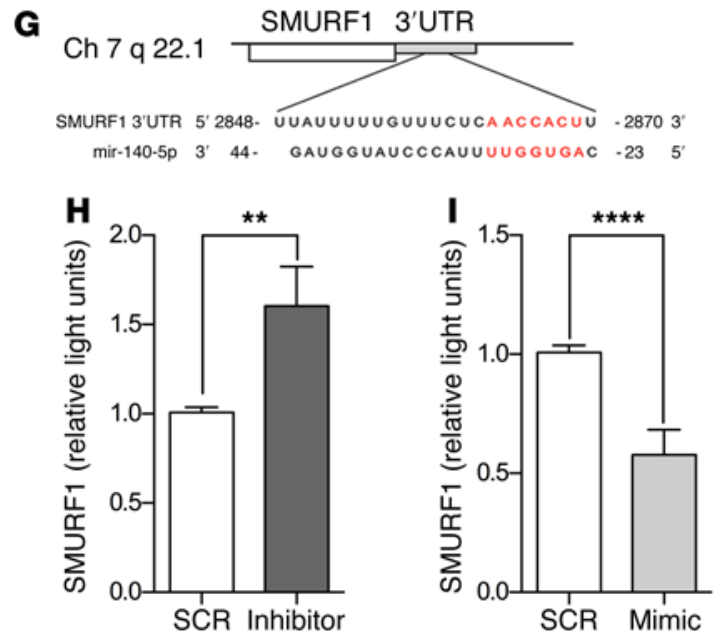
Figure 6. miR-140-5p regulates key mediators of PAH pathology. (A) Predicted PH-related direct targets of miR-140-5p. (B and C) qPCR (B) and protein expression by Western blot (C) showing expression of miR-140$5 p$ targets ALK5, PDGFRA, and SMURF1 in PASMCs at 72 hours following transfection with miR-140-5p mimic (M), miR-140-5p inhibitor (I), or scramble control (S) (B: $n=6, \mathbf{C}: n=5, \mathbf{B}$ and C: ${ }^{*} P<0.05$; ${ }^{*} P<0.01$; ${ }^{* *} P<0.001$; ${ }^{* * *} P<0.0001$, 1-way ANOVA with Tukey's post-test correction, mean \pm SEM). (D) Whole lung levels of SMURF1 protein correlate inversely with miR-140-5p ( $n=38, P<0.05, r=-0.36$, Spearman rank). (E) Increased whole blood levels of SMURF1 mRNA were observed in patients with IPAH when compared with HV (IPAH $n=20, \mathrm{HV} n=16$, ${ }^{*} P<0.05$, unpaired 2-tailed Student's $t$ test, mean \pm SEM). P, prevention; T, therapeutic. (F) Representative photomicrographs demonstrating immunoreactivity of SMURF1 in endothelial and SMCs of pulmonary vascular lesions in the transplanted lungs of patients with BMPR2 mutation-associated heritable $\mathrm{PAH}$, associated $\mathrm{PAH}$, and non-PAH control lung (photomicrographs representative of $n=26$ : APAH $n=8$, IPAH $n=8$, FPAH, $n=6$, control $n=4$ ). Original magnification, $\times 200$ (hereditary PAH and control); $\times 400$ (associated PAH). Scale bars: $50 \mu \mathrm{m}$. (C) Chromosomal location of putative miR-140-5p-binding site in the $3^{\prime}$ UTR of SMURF1. (H and I) Transfection of PASMCs with miR-140-5p inhibitor $(\mathbf{H})$ and mimic (I) alter luciferase activity of SMURF1 $3^{\prime}$ UTR construct at 24 hours ( $H$ and $\mathbf{I}: n=4,{ }^{* *} P<0.01$, ${ }^{* * * *} P<0.0001,2$-tailed Mann-Whitney $U$ test, mean \pm SEM).

improved disease phenotype in experimental PAH via modulation of key cellular targets. Network analysis identified the E3 ubiquitin ligase SMURF1 as a potential central regulator of PH-related miR-140-5p targets and BMP signaling. SMURF1 whole blood mRNA and pulmonary vascular SMURF1 protein expression were increased in patients with $\mathrm{PAH}$, providing further evidence of a role in human disease pathology. The critical importance of SMURF1 in the development of PAH was demonstrated by genetic deletion of Smurf1, which provided an allele-dependent protection from experimental disease. Thus, the interaction between miR-140-5p and SMURF1 represents a mechanism by which BMP signaling may be reduced in patients with PAH without an identifiable BMPR2 mutation, and inhibition of SMURF1 provides a mechanism by which BMP signaling may be augmented for therapeutic benefit in patients with PAH.

Recent years have seen significant advances in the treatment of PAH; however, there remains no pharmacological cure for this devastating disease $(41,42)$. Therapeutic options are limited to vasodilatation via the prostacyclin, endothelin, or nitric oxide pathways; however, proliferative remodeling continues, and normalization of pulmonary vascular resistance with long-term improvement is rarely achieved. Targeting the proliferation that drives disease pathology is an attractive concept. Excellent proof of concept for this strategy is provided by the positive preclinical $(43)$ and clinical $(44,45)$ hemodynamic effects of imatinib, although clinical approval was limited by an adverse side effect profile.

Impaired BMP signaling, through gene mutation or environmental insult, is a consistent finding in human $(7-9,46)$ and experimental PAH $(10,11)$. BMPR2 mutation results in haploinsufficiency of the receptor and reduced pathway activity; however, penetrance is incomplete, and not all patients with the mutation have disease. It has been proposed that in the presence of a BMPR2 mutation, further pathway suppression is required for disease development, and in the absence of BMPR2 mutation, the mechanism by which signaling is reduced remains unclear.
Genetic restoration of BMPR2 attenuates both chronic hypoxia and MCT-induced PAH $(23,24)$. The selective BMP agonist BMP9 (15) and drugs that nonspecifically enhance BMP signaling, such as FK506 (26), chloroquine (27), and paclitaxel (28), have shown beneficial effects in preclinical models. Early studies of FK506 have shown clinical effect during compassionate use in patients with PAH. An alternative approach to enhancing BMP signaling may be through inhibition of negative regulators of the pathway (27). SMURF1 negatively regulates BMP signaling through ubiquitination and proteasomal degradation of BMPR2 (47), type I BMP receptors, and downstream signaling mediators (48). Through human array data and bioinformatic prediction, we have identified miR-140-5p and SMURF1 as 2 means of modulating BMP signaling in PAH.

miR-140-5p is considered a tumor suppressor miR, downregulation of which has been identified in a number of neoplastic conditions (32-35). Loss of miR-140-5p led to increased cellular proliferation and migration through derepression of previously validated targets, including PDGFRA (49), type 1 IGF receptor (IGF1R) (33), ALK5, and FGF2 (32). miR-140-5p is known to play a critical role in bone and skeletal development, mediating downstream effects of IL- $1 \beta$ and TGF- $\beta$ through interactions with ADAM metallopeptidase with thrombospondin type 1 motif 5 (ADAMTS5) (50) and SP1 transcription factor (SP1) (51). Furthermore, the rs7205289 single-nucleotide polymorphism is associated with nonsyndromic cleft palate and alters processing of premiR-140, resulting in reduced expression of miR-140-5p.

Locked nucleic acid structures are deliverable and stable in the circulation, providing an effective means of anti-miR or antagomiR therapy. The clinical application of such miR-based therapies has shown significant promise (52). In 3 experimental models of PAH, we demonstrate that increased miR-140-5p prevents disease development and attenuates the progression of established disease. However, administration of miR-140-5p mimic did not fully reverse established disease. This may be attributable to a number of factors: the disease phenotype developed in therapeutic studies was severe; nebulized delivery of miR-140-5p provided incomplete restoration of whole lung levels compared with those in untreated animals; and modulation of a large number of mRNA by delivery of a single miR will result in repression of those with both positive and negative effects on disease phenotype. Furthermore, the development of pre-miR or miR mimic technology has lagged behind that of anti-miR therapy (53), and for these reasons, we sought to identify molecules downstream of miR-140-5p as therapeutic targets. We demonstrate in vitro that miR-140-5p regulates expression of SMURF1 through direct interaction with the 3' UTR and that both miR-140-5p and SMURF1 modulate BMP signaling in PASMCs.

SMURF1 is a homologous to the E6-AP carboxyl terminus-type (HECT-type) ubiquitin ligase that acts as a negative regulator of BMP signaling via ubiquitination and subsequent proteasomal degradation of SMAD1, SMAD5 (48), and BMPR2 (47). SMURF1 regulates cell migration and adhesion modulating signaling downstream of BMP $(47,48)$, TGF- $\beta$, and IFN- $\gamma(54)$, and amplification of SMURF1 has been observed and implicated in human pancreatic cancer (55). Expression of SMURF1 is increased in animal models of PAH (47). We demonstrate 
A
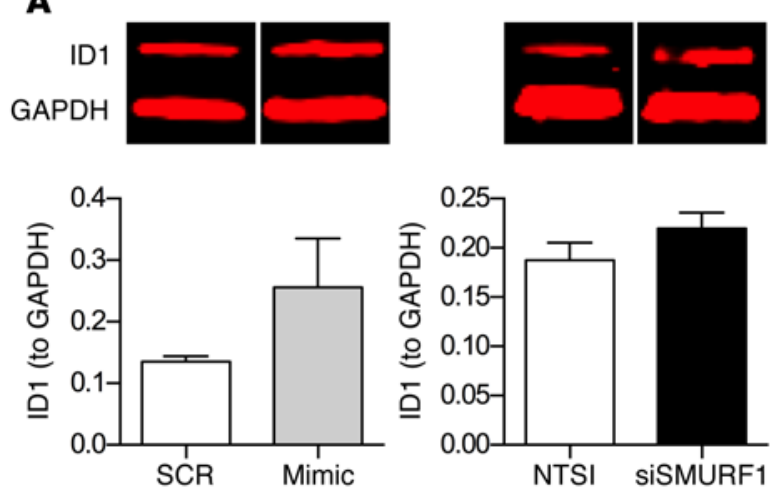

D

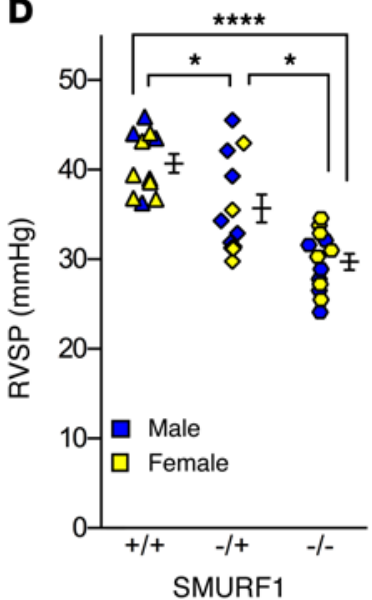

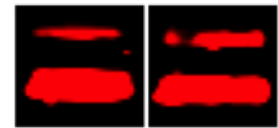

SISMURF1
B

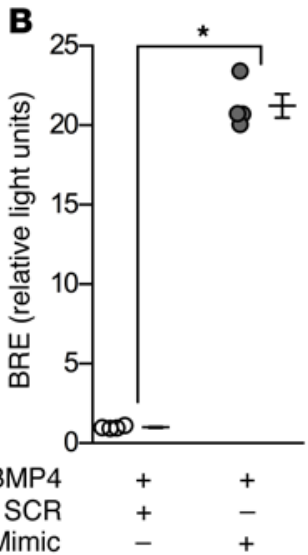

E

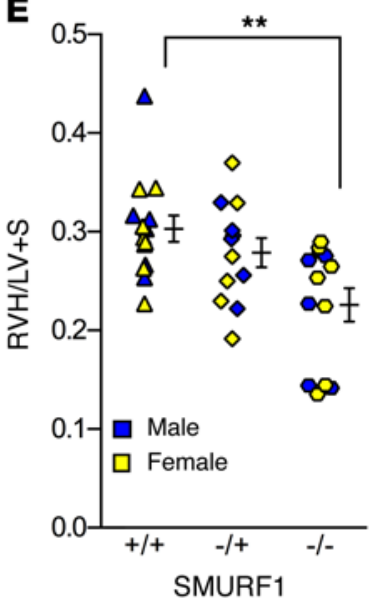

C

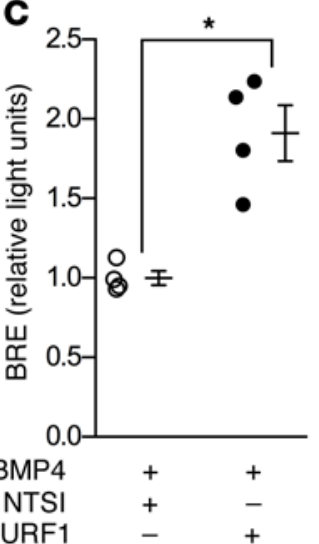

$\mathbf{F}$

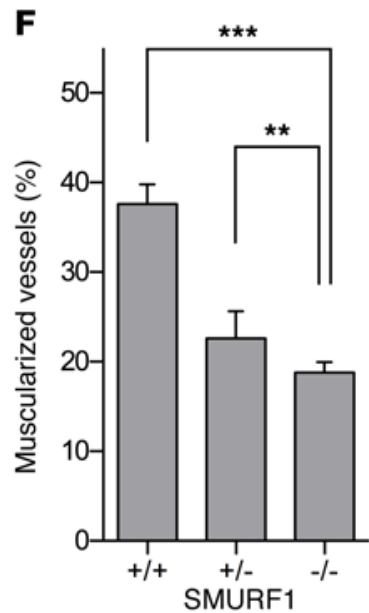

Figure 7. miR-140-5p and SMURF1 modulate BMP signaling in vitro, and SMURF1 is critical to the development of experimental PAH. (A-C) miR-140-5p mimic and SMURF1 siRNA augment BMP signaling in vitro. (A) PASMCs were transfected with SCR and miR-140-5p mimic or NTsiRNA and SMURF1 siRNA prior to stimulation with BMP4. At 72 hours, expression of native ID1 protein was increased in miR-140-5p mimic- and SMURF1 siRNA-treated cells $(n=4$, mean \pm SEM). (B) PASMCs were cotransfected with BRE construct and SCR or miR-140-5p prior to stimulation with BMP4. At 24 hours, BRE activity was increased in miR-140-5p mimic-treated cells. (C) PASMCs were cotransfected with BRE construct and NTsiRNA or SMURF1 siRNA prior to stimulation with BMP4. At 24 hours, BRE activity was increased in SMURF1 siRNA-treated cells. (B and $\mathbf{C}: n=4,{ }^{*} P<0.05,2$-tailed Mann-Whitney $U$ test, mean \pm SEM). (D-F) Genetic ablation of SMURF1 confers protection from PAH in the SuHx mouse model. (D) RVSP at week 3. (E) RVH at week 3. (F) Vessel muscularization at week 3. (D-F: $n=11-13$ per group, ${ }^{*} P<0.05 ;{ }^{* *} P<0.01$; ${ }^{* * *} P<0.001$, ${ }^{* * *} P<0.0001,1$-way ANOVA with Tukey's post-test correction, mean \pm SEM).

increased expression of SMURF1 mRNA in whole blood and SMURF1 protein immunoreactivity within pulmonary vascular lesions from patients with PAH. Consistent with our clinical findings and bioinformatic predictions, reduced levels of miR140-5p lead to derepression of SMURF1 in vitro, and both miR140-5p mimic and inhibitor alter expression of SMURF1 (mRNA, protein, and 3' UTR reporter construct) in PASMCs, validating the interaction in a disease-relevant cell.

SMURF1 is a key negative regulator of the BMP pathway, and the effect of small alterations in cellular levels has an amplified effect on downstream signaling and cell phenotype. The critical importance of SMURF1 to the development of experimental PAH is demonstrated by the allele-dependent protection provided by genetic deletion in the SuHx model.

The identification of small molecule inhibitors of SMURF1 (56) offers the opportunity to target negative regulators of BMP signaling as a strategy for antiproliferative treatments of PAH. Furthermore, our data suggest that whole blood miR-140-5p and SMURF1 levels may be used to identify patients with abnormal signaling in this pathway.

\section{Methods}

\section{Patient groups and study design}

For TaqMan miR array studies, RNA was extracted from individual patient samples and quantified as described below. Samples were pooled by clinical diagnosis ( $n=4$ per group: IPAH, CTD-PH, CTEPH). The 2 control groups used were as follows: group 1: HV; group 2: patients with CTD referred for investigation for $\mathrm{PH}$, but found to have normal pulmonary hemodynamics ( $n=4$ per group: HV and CTD). Validation of array findings was undertaken by 2 means: single-sample qPCR assay of individual patient samples from those used in array and single-sample qPCR assay of individual patient samples from a second cohort of patients with IPAH and HV. Clinical details of patients included are provided in Supplemental Tables 1 and 2.

Examination of SMURF1 expression in explanted lungs was undertaken using patient samples from the Papworth NHS Foundation Trust Hospital Tissue Bank samples. PAH lung and control tissues were formalin-fixed, paraffin-processed wax block samples that were returned to the Tissue Bank after sectioning (associated PAH, $n=8$; idiopathic PAH, $n=8$; familial PAH, $n=6$, and control, $n=4$ ). 
Control tissue comprised cases of unused donor lung and tissue from lobes of lung clear of tumor taken from pneumonectomy specimens. Lungs were inflated using a pressure-limited pump with peribronchial neutral-buffered $10 \%$ formalin solution (Genta Medical) and fixed for at least 24 hours; sections were examined macroscopically by a pathologist, and representative tissue blocks were taken for microscopic examination.

\section{Whole blood RNA extraction and quantification}

Blood samples were drawn in Tempus whole blood tubes (Applied Biosystems) at the time of diagnosis from patients with $\mathrm{PH}$ and matched control participants. Total RNA (miR and mRNA) was extracted using an miR/mRNA extraction kit (Promega) on the Maxwell platform (Promega). mRNA purity and concentration were assessed by Nanodrop (Thermo Fisher Scientific).

\section{TaqMan low-density array of miR}

miRs were reverse transcribed to single-stranded cDNA using the TaqMan microRNA Reverse Transcription Kit (Invitrogen). Reverse transcribed products from individual samples were pooled and expression quantified on TaqMan Human MicroRNA V2.0 A (Invitrogen). miR levels were normalized to U6 and analyzed by fold change in HTqPCR (57).

\section{TaqMan PCR}

Expression of miR and mRNA was determined by PCR using TaqMan Small RNA Assay (U6, 001973 and miR-140-5p 001187, Invitrogen) or TaqMan Gene Expression Assay (18S, HS03003631_g1; ID1, Hs03676575_s1; SMURF1, Hs00410929_m1; RUNX2, HS00231692_ $\mathrm{m} 1$, Invitrogen). miR and mRNA levels were normalized to U6 and $18 \mathrm{~S}$, respectively, for quantification using the $\Delta \Delta \mathrm{Ct}$ comparative quantification method as previously described (13).

\section{Cell culture and phenotypic assays}

Human PASMCs (Lonza) were maintained as previously described (58). Before stimulation, PASMCs were synchronized by incubating in DME medium containing PSA Solution (Invitrogen) and $0.2 \%$ fetal bovine serum for 48 hours. Cell proliferation was quantified by DNA staining (LI-COR Biosciences) and migration using a Boyden chamber assay (59). Where stated, cells were transfected with miR-140-5p mimic, inhibitor, or scramble control (Invitrogen) or SMURF1 siRNA (Dharmacon) using HappyFect (Tecra), and stimulated with $20 \mathrm{ng} / \mathrm{ml}$ recombinant human PDGFBB or $10 \mathrm{ng} / \mathrm{ml}$ BMP4 (R\&D Systems) in DME medium containing PSA solution (Invitrogen) in $0.2 \%$ fetal bovine serum.

\section{Luciferase}

PASMCs were transfected with 100 ng of plasmid vector (SMURF1 3' UTR, HmiT055054-MT01, Genecopia; pGL4 luc2p/ID1/Hygro, Promega pRL-TK, Promega) with miR-140-5p mimic, inhibitor, or scramble control (Invitrogen) or SMURF1 siRNA (Dharmacon) per the manufacturer's instructions. Twenty-four hours after transfection, cells were harvested and lysed and luciferase reporter activities measured using the Dual-Glo Luciferase Assay System (Promega). Firefly luciferase activity was normalized to Renilla luciferase activity. For BMP activity assays, cells were stimulated with BMP4 (R\&D Systems) at $50 \mathrm{ng} / \mathrm{ml}$ in DME medium containing PSA solution (Invitrogen) in $0.2 \%$ fetal bovine serum.

\section{Western immunoblotting}

Sample $(40 \mu \mathrm{g})$ was loaded on a $4 \%$ to $12 \%$ Bis-Tris NuPage gel, run under reducing conditions in MOPS running buffer (Invitrogen), and transferred to a nitrocellulose membrane (Invitrogen). Membranes were blocked (blocking buffer, LI-COR Biosciences) for 90 minutes at room temperature, then incubated with ALK5 (1:500, ab31013, Abcam), ID1 (1:1000, clone 195-14, CalBioreagents), PDGFRA (1:250, ab61219, Abcam), SMURF1 (1:250, Ab57573, Abcam), or GAPDH antibody (1:1000, 14C10, Cell Signaling Technology) overnight at $4^{\circ} \mathrm{C}$ with Quick Western IRDye (LI-COR Biosciences) prior to a reading at $700 \mathrm{~nm}$ on an Odyssey SA imaging system (LI-COR Biosciences).

\section{Target prediction}

miR target prediction was performed using the TargetScan 6.2 (conserved) algorithm (39), which identifies mRNA transcripts with conserved complementarity to the miR "seed sequence" (nucleotides 2-7) and biologically validated interactions. Functional annotation was performed using the Database for Annotation, Visualization and Integrated Discovery (DAVID) (60).

PH-related miR-140-5p targets were identified using a curated set of PH-relevant genes (Supplemental Table 6). These genes were selected based on their cooccurrence with the search term "pulmonary hypertension" in the PubMed database (37). Interactions between network genes were annotated according to a list of protein-protein interactions (61-63), protein-DNA interactions (64), and kinase-substrate interactions (65) drawn from consolidated databases.

\section{Animal studies}

MCT rat studies. PAH was induced in male Sprague-Dawley rats (Charles River Laboratories) weighing 200-210 g by a single subcutaneous injection of MCT (Sigma-Aldrich) at $60 \mathrm{mg} / \mathrm{kg}$. For time-course experiments, animals were sacrificed at days 2, 7, and 28. Preventative intervention was performed at days 0,7 , and 14 and animals sacrificed at day 21. Therapeutic intervention was performed at days 21 and 28 and animals sacrificed at day 35 .

Sugen hypoxia rat studies. PAH was induced in male Wistar Kyoto (Harlan) rats weighing 200-230 g by a single subcutaneous injection of SU5416 (Tocris) at $20 \mathrm{mg} / \mathrm{kg}$, followed by housing at $10 \% \mathrm{O}_{2}$ and $5 \% \mathrm{CO}_{2}$ for 3 weeks and normoxia for remaining weeks. For timecourse experiments, animals were sacrificed at 3, 5, 8, and 14 weeks. Therapeutic experimentation was undertaken at 8,9 , and 10 weeks, with sacrifice at 11 weeks.

SuHx Smurf1 mouse studies. Smurf1 KO (Smurf1 $\left.1^{-/}\right)$mice were obtained under license from Y. Zhang (Laboratory of Cellular and Molecular Biology, Center for Cancer Research, National Cancer Institute, Bethesda, Maryland, USA) (66). Smurf1 ${ }^{-/-}$mice were crossed with WT C57BL/6 mice to generate SMURF1 heterozygotes $\left(\right.$ Smurf1 $\left.1^{+/}\right)$, which were subsequently crossed to generate Smurf1 $1^{-/}$and littermate controls. Mice were administered SU5416 (Sigma-Aldrich) at $20 \mathrm{mg} /$ $\mathrm{kg}$ once weekly under normoxia or hypoxia as described previously (67). At study termination, mice were sacrificed and PAH pathology was assessed as described previously (12).

Where stated, in vivo transfection was performed with $20 \mu \mathrm{M}$ of miR140-5p mimic or scramble control (Invitrogen) using Invivofectamine 2 (Invitrogen) delivered by MicroSprayer Aerosolizer (Penn-Century Inc.). Transfection efficiency was assessed by qPCR and tissue distribution assessed by delivery of Cy3-labeled scramble control ( $20 \mu \mathrm{M}$, Invitrogen). 
Animals were monitored for signs of distress and right heart failure, defined by loss of body weight (either $>5 \% / 24$ hours or $>10 \% / 48$ hours), lethargy, and respiratory distress, as previously described $(68,69)$.

Echocardiography, cardiac catheterization, and RVH. Echocardiography was performed at baseline and sacrifice in prevention studies and at baseline, pretreatment, and sacrifice in therapeutic studies (Vivo 770, Visualsonics). Closed chest cardiac catheterization (Millar) was performed at sacrifice in time-course and intervention studies. Echocardiography, cardiac catheterization, and measurement of RVH were performed as previously described (59).

\section{Rodent $\mathrm{miR} / \mathrm{mRNA}$ extraction and quantification}

Total RNA (miR and mRNA) was extracted using an miR/mRNA extraction kit (Promega) on the Maxwell platform (Promega). mRNA purity and concentration were assessed by Nanodrop (Thermo Fisher Scientific). PCR was performed as described above.

\section{Rodent protein extraction and quantification}

Whole-lung segments were snap-frozen, then ground under liquid nitrogen. Powder was stored at $-80^{\circ} \mathrm{C}$ prior to extraction with T-PER Tissue Protein Extraction Reagent (Thermo Scientific). Western immunoblotting was performed as described above.

\section{Immunohistochemistry}

Lung tissue was harvested, processed. and stained as previously described (59). Paraffin-embedded sections $(5 \mu \mathrm{m})$ were stained for Alcian blue elastin van Gieson (EVG) and H\&E. Immunohistochemical staining used antibodies against SMA (M0851; Dako) to visualize SMCs and von Willebrand Factor (vWF; A0082, Dako) to visualize endothelial cells. SMURF1 (ab57573, Abcam) was used to localize protein expression within pulmonary vascular lesions. Mouse anti-human PCNA antibody (M0879; Dako) was used to assess proliferation. Species-appropriate biotinylated secondary antibody (1:200) with Avidin Biotin Enzyme Complex (Vectastain kit, Vector Laboratories) or species-appropriate florescent antibody was used (Alexa Fluor 488 dye, Thermo Fisher Scientific) was used. Microscopy was performed on the Axiocam 506 Color System (Zeiss) and analysis undertaken in Zen 2 Blue Edition (Zeiss).

\section{Quantification of pulmonary vascular remodeling}

Pulmonary vascular remodeling was quantified by assessing the degree of muscularization and the percentage of affected pulmonary arteries and arterioles in 3 groups based on vessel size: small pulmonary arterioles with a diameter less than $50 \mu \mathrm{m}$, medium pulmonary arteries with a range in diameter from 51 to $100 \mu \mathrm{m}$, and large pulmonary arteries with a diameter greater than $100 \mu \mathrm{m}(13,59)$.

\section{Statistics}

Statistical analysis was performed as stated in Prism 6.0h for Macintosh (GraphPad Software). Normality of data distribution was assessed by Kolmogorov-Smirnov test and differences between groups determined by 2-tailed Student's $t$ test, Mann-Whitney $U$ test, or ANOVA with post-test correction as appropriate. $P<0.05$ was considered statistically significant.

\section{Study approval}

Human samples. All subjects gave informed written consent, and the study was approved by the Yorkshire and the Humber - Sheffield Research Ethics Committee and the Sheffield Teaching Hospitals Foundation Trust Observational Cardiovascular Biobank (08/H1308/193 and STH15222).

Animal studies. All animal experiments were approved by the University of Sheffield Project Review Committee and conformed to United Kingdom Home Office ethical guidelines.

\section{Author contributions}

AMKR designed research studies, conducted experiments, acquired data, analyzed data, and wrote the manuscript. NDA designed research studies, conducted experiments, acquired data, and analyzed data. JAP designed research studies, conducted experiments, acquired data, analyzed data, and wrote the manuscript. JI designed research studies, conducted experiments, and acquired data. LC designed research studies, conducted experiments, acquired data, and analyzed data. RMHA designed research studies, conducted experiments, acquired data, and analyzed data. SGG designed research studies, conducted experiments, acquired data, and analyzed data. MS designed research studies, conducted experiments, and acquired data. NWM designed research studies and wrote the manuscript. MT designed research studies. SEF wrote the manuscript. DJR designed research studies and wrote the manuscript. AL designed research studies, acquired data, analyzed data, and wrote the manuscript.

\section{Acknowledgments}

Funding for this study was provided by a Medical Research Council Clinical Research training fellowship (to A.M.K. Rothman: MR/K002406/1); a Medical Research Council Career Development award (to A. Lawrie: G0800318); a British Heart Foundation Senior Basic Science fellowship (to A. Lawrie: FS/13/48/30453); and a British Heart Foundation Project grant (PG/11/11629288). The collection of patient blood samples was coordinated through the Sheffield National Institute for Health Research (NIHR) Clinical Research Facility. The Cambridge NIHR Biomedical Research Centre contributed to infrastructure support.

Address correspondence to: Allan Lawrie, Department of Infection, Immunity and Cardiovascular Disease, University of Sheffield, Medical School, Beech Hill Rd., Sheffield, South Yorkshire S10 2RX, United Kingdom. Phone: 44.0.1142261432; E-mail: a.lawrie@sheffield.ac.uk.

Matthew Thomas's present address is: Astra Zeneca R\&D, Respiratory, Inflammation, and Autoimmunity (RIA), Gothenburg, Sweden.
1. Machado RD, et al. Genetics and genomics of pulmonary arterial hypertension. JAm Coll Cardiol. 2009;54(1 suppl):S32-S42.

2. Hassoun PM, et al. Inflammation, growth factors, and pulmonary vascular remodeling. J Am Coll
Cardiol. 2009;54(1 suppl):S10-S19.

3. Tuder RM, et al. Relevant issues in the pathology and pathobiology of pulmonary hypertension. J Am Coll Cardiol. 2013;62(25 suppl):D4-D12. 4. Vonk Noordegraff A, et al. Right heart adap- tation to pulmonary arterial hypertension: physiology and pathobiology. J Am Coll Cardiol. 2013;62(25 suppl):D22-D33.

5. Galiè N, et al. Updated treatment algorithm of pulmonary arterial hypertension. JAm Coll Car- 
diol. 2013;62(25 suppl):D60-D72.

6. Galiè N, et al. 2015 ESC/ERS Guidelines for the diagnosis and treatment of pulmonary hypertension: The Joint Task Force for the Diagnosis and Treatment of Pulmonary Hypertension of the European Society of Cardiology (ESC) and the European Respiratory Society (ERS): Endorsed by: Association for European Paediatric and Congenital Cardiology (AEPC), International Society for Heart and Lung Transplantation (ISHLT). Eur Heart J. 2016;37(1):67-119.

7. Deng Z, et al. Familial primary pulmonary hypertension (gene PPH1) is caused by mutations in the bone morphogenetic protein receptor-II gene. Am J Hum Genet. 2000;67(3):737-744.

8. Thomson JR, et al. Sporadic primary pulmonary hypertension is associated with germline mutations of the gene encoding BMPR-II, a receptor member of the TGF- $\beta$ family. JMed Genet. 2000;37(10):741-745.

9. Atkinson C, et al. Primary pulmonary hypertension is associated with reduced pulmonary vascular expression of type II bone morphogenetic protein receptor. Circulation. 2002;105(14):1672-1678.

10. Takahashi H, et al. Downregulation of type II bone morphogenetic protein receptor in hypoxic pulmonary hypertension. Am J Physiol Lung Cell Mol Physiol. 2006;290(3):L450-L458.

11. Morty RE, et al. Dysregulated bone morphogenetic protein signaling in monocrotaline-induced pulmonary arterial hypertension. Arterioscler Thromb Vasc Biol. 2007;27(5):1072-1078.

12. Ciuclan L, et al. A novel murine model of severe pulmonary arterial hypertension. Am J Respir Crit Care Med. 2011;184(10):1171-1182.

13. Lawrie A, et al. Paigen diet-fed apolipoprotein $\mathrm{E}$ knockout mice develop severe pulmonary hypertension in an interleukin-1-dependent manner. Am J Pathol. 2011;179(4):1693-1705.

14. Burton VJ, Ciuclan LI, Holmes AM, Rodman DM, Walker C, Budd DC. Bone morphogenetic protein receptor II regulates pulmonary artery endothelial cell barrier function. Blood. 2011;117(1):333-341.

15. Long L, et al. Selective enhancement of endothelial BMPR-II with BMP9 reverses pulmonary arterial hypertension. Nat Med. 2015;21(7):777-785.

16. Teichert-Kuliszewska K, et al. Bone morphogenetic protein receptor-2 signaling promotes pulmonary arterial endothelial cell survival: implications for loss-of-function mutations in the pathogenesis of pulmonary hypertension. Circ Res. 2006;98(2):209-217.

17. Morrell NW, et al. Altered growth responses of pulmonary artery smooth muscle cells from patients with primary pulmonary hypertension to transforming growth factor-beta(1) and bone morphogenetic proteins. Circulation. 2001;104(7):790-795.

18. Yang X, et al. Dysfunctional Smad signaling contributes to abnormal smooth muscle cell proliferation in familial pulmonary arterial hypertension. Circ Res. 2005;96(10):1053-1063.

19. Zhang S, et al. Bone morphogenetic proteins induce apoptosis in human pulmonary vascular smooth muscle cells. Am J Physiol Lung Cell Mol Physiol. 2003;285(3):L740-L754.

20. Hong KH, et al. Genetic ablation of the BMPR2 gene in pulmonary endothelium is sufficient to predispose to pulmonary arterial hypertension. Circulation. 2008;118(7):722-730.

21. West J, et al. Pulmonary hypertension in transgenic mice expressing a dominant-negative BMPRII gene in smooth muscle. Circ Res. 2004;94(8):1109-1114.

22. Zaidi SH, You XM, Ciura S, Husain M, Rabinovitch $\mathrm{M}$. Overexpression of the serine elastase inhibitor elafin protects transgenic mice from hypoxic pulmonary hypertension. Circulation. 2002;105(4):516-521.

23. Reynolds AM, et al. Bone morphogenetic protein type 2 receptor gene therapy attenuates hypoxic pulmonary hypertension. Am J Physiol Lung Cell Mol Physiol. 2007;292(5):L1182-L1192.

24. Reynolds AM, Holmes MD, Danilov SM, Reynolds PN. Targeted gene delivery of BMPR2 attenuates pulmonary hypertension. Eur Respir J. 2012;39(2):329-343.

25. Nickel NP, et al. Elafin Reverses Pulmonary Hypertension via Caveolin-1-Dependent Bone Morphogenetic Protein Signaling. Am J Respir Crit Care Med. 2015;191(11):1273-1286.

26. Spiekerkoetter E, et al. FK506 activates BMPR2, rescues endothelial dysfunction, and reverses pulmonary hypertension. JClin Invest. 2013;123(8):3600-3613.

27. Long L, et al. Chloroquine prevents progression of experimental pulmonary hypertension via inhibition of autophagy and lysosomal bone morphogenetic protein type II receptor degradation. Circ Res. 2013;112(8):1159-1170.

28. Savai R, et al. Pro-proliferative and inflammatory signaling converge on FoxO1 transcription factor in pulmonary hypertension. Nat Med. 2014;20(11):1289-1300.

29. Rothman AM, Chico TJ, Lawrie A. MicroRNA in pulmonary vascular disease. Prog Mol Biol Transl Sci. 2014;124:43-63.

30. Schlosser K, Taha M, Deng Y, Jiang B, Stewart DJ. Discordant regulation of microRNA between multiple experimental models and human pulmonary hypertension. Chest. 2015;148(2):481-490.

31. Yang J, et al. Sildenafil potentiates bone morphogenetic protein signaling in pulmonary arterial smooth muscle cells and in experimental pulmonary hypertension. Arterioscler Thromb Vasc Biol. 2013;33(1):34-42.

32. Yang H, Fang F, Chang R, Yang L. MicroRNA140-5p suppresses tumor growth and metastasis by targeting TGFBR1 and FGF9 in hepatocellular carcinoma. Hepatology. 2013;58(1):205-217.

33. Yuan Y, Shen Y, Xue L, Fan H. miR-140 suppresses tumor growth and metastasis of non-small cel lung cancer by targeting insulin-like growth factor 1 receptor. PLoS One. 2013;8(9):e73604.

34. Li Q, Yao Y, Eades G, Liu Z, Zhang Y, Zhou Q. Downregulation of miR-140 promotes cancer stem cell formation in basal-like early stage breast cancer. Oncogene. 2014;33(20):2589-2600.

35. Zhang Y, Eades G, Yao Y, Li Q, Zhou Q. Estrogen receptor $\alpha$ signaling regulates breast tumorinitiating cells by down-regulating miR-140 which targets the transcription factor SOX2. J Biol Chem. 2012;287(49):41514-41522.

36. Courboulin A, et al. Role for miR-204 in human pulmonary arterial hypertension. JExp Med.
2011;208(3):535-548.

37. Parikh VN, et al. MicroRNA-21 integrates pathogenic signaling to control pulmonary hypertension: results of a network bioinformatics approach. Circulation. 2012;125(12):1520-1532.

38. Bertero T, et al. Systems-level regulation of microRNA networks by miR-130/301 promotes pulmonary hypertension. JClin Invest. 2014;124(8):3514-3528

39. Friedman RC, Farh KK, Burge CB, Bartel DP. Most mammalian mRNAs are conserved targets of microRNAs. Genome Res. 2009;19(1):92-105.

40. Yang J, et al. Mutations in bone morphogenetic protein type II receptor cause dysregulation of Id gene expression in pulmonary artery smooth muscle cells: implications for familial pulmonary arterial hypertension. Circ Res. 2008;102(10):1212-1221.

41. Hurdman J, et al. ASPIRE registry: assessing the Spectrum of Pulmonary hypertension Identified at a REferral centre. Eur Respir J 2012;39(4):945-955.

42. Benza RL, Miller DP, Barst RJ, Badesch DB, Frost AE, McGoon MD. An evaluation of long-term survival from time of diagnosis in pulmonary arterial hypertension from the REVEAL Registry. Chest. 2012;142(2):448-456.

43. Schermuly RT, et al. Reversal of experimental pulmonary hypertension by PDGF inhibition. JClin Invest. 2005;115(10):2811-2821.

44. Ghofrani HA, Seeger W, Grimminger F. Imatinib for the treatment of pulmonary arterial hypertension. N Engl JMed. 2005;353(13):1412-1413.

45. Hoeper MM, et al. Imatinib mesylate as add-on therapy for pulmonary arterial hypertension: results of the randomized IMPRES study. Circulation. 2013;127(10):1128-1138.

46. Aldred MA, et al. Somatic chromosome abnormalities in the lungs of patients with pulmonary arterial hypertension. Am J Respir Crit Care Med. 2010;182(9):1153-1160.

47. Murakami K, et al. Smurf1 ubiquitin ligase causes downregulation of BMP receptors and is induced in monocrotaline and hypoxia models of pulmonary arterial hypertension. Exp Biol Med (Maywood). 2010;235(7):805-813.

48. Zhu H, Kavsak P, Abdollah S, Wrana JL, Thomsen GH. A SMAD ubiquitin ligase targets the BMP pathway and affects embryonic pattern formation. Nature. 1999;400(6745):687-693.

49. Eberhart JK, et al. MicroRNA Mirn140 modulates Pdgf signaling during palatogenesis. Nat Genet. 2008;40(3):290-298

50. Miyaki S, et al. MicroRNA-140 is expressed in differentiated human articular chondrocytes and modulates interleukin-1 responses. Arthritis Rheum. 2009;60(9):2723-2730.

51. Yang J, et al. MiR-140 is co-expressed with Wwp2-C transcript and activated by Sox 9 to target Sp1 in maintaining the chondrocyte proliferation. FEBS Lett. 2011;585(19):2992-2997.

52. Janssen HL, et al. Treatment of HCV infection by targeting microRNA. $N$ Engl J Med 2013;368(18):1685-1694

53. van Rooij E, Purcell AL, Levin AA. Developing microRNA therapeutics. Circ Res. 2012;110(3):496-507.

54. Cao Y, Zhang L. A Smurf1 tale: function and 


\section{RESEARCH ARTICLE}

regulation of an ubiquitin ligase in multiple cellular networks. Cell Mol Life Sci. 2013;70(13):2305-2317.

55. Kwei KA, et al. SMURF1 amplification promotes invasiveness in pancreatic cancer. PLoS One. 2011;6(8):e23924.

56. Cao Y, et al. Selective small molecule compounds increase BMP-2 responsiveness by inhibiting Smurf1-mediated Smad1/5 degradation. Sci Rep. 2014;4:4965.

57. Dvinge H, Bertone P. HTqPCR: high-throughput analysis and visualization of quantitative real-time PCR data in R. Bioinformatics. 2009;25(24):3325-3326.

58. Lawrie A, et al. Evidence of a role for osteoprotegerin in the pathogenesis of pulmonary arterial hypertension. Am J Pathol. 2008;172(1):256-264.

59. Hameed AG, et al. Inhibition of tumor necrosis factor-related apoptosis-inducing ligand (TRAIL) reverses experimental pulmonary hypertension.
JExp Med. 2012;209(11):1919-1935.

60. Huang da W, Sherman BT, Lempicki RA. Systematic and integrative analysis of large gene lists using DAVID bioinformatics resources. Nat Protoc. 2009;4(1):44-57.

61. Rual JF, et al. Towards a proteome-scale map of the human protein-protein interaction network. Nature. 2005;437(7062):1173-1178.

62. Aranda B, et al. The IntAct molecular interaction database in 2010. Nucleic Acids Res. 2010;38(Database issue):D525-D531.

63. Licata L, et al. MINT, the molecular interaction database: 2012 update. Nucleic Acids Res. 2012;40(Database issue):D857-D861.

64. Matys V. TRANSFAC(R): transcriptional regulation, from patterns to profiles. Nucleic Acids Res. 2003;31(1):374-378.

65. Diella F, Gould CM, Chica C, Via A, Gibson TJ. Phospho.ELM: a database of phosphorylation sites - update 2008. Nucleic Acids Res.
2008;36(Database issue):D240-D244.

66. Yamashita M, et al. Ubiquitin ligase Smurf1 controls osteoblast activity and bone homeostasis by targeting MEKK2 for degradation. Cell. 2005;121(1):101-113.

67. Ciuclan L, et al. Imatinib attenuates hypoxiainduced pulmonary arterial hypertension pathology via reduction in 5-hydroxytryptamine through inhibition of tryptophan hydroxylase 1 expression. Am J Respir Crit Care Med. 2013;187(1):78-89.

68. Merklinger SL, Jones PL, Martinez EC, Rabinovitch M. Epidermal growth factor receptor blockade mediates smooth muscle cell apoptosis and improves survival in rats with pulmonary hypertension. Circulation. 2005;112(3):423-431.

69. de Man FS, et al. Bisoprolol delays progression towards right heart failure in experimental pulmonary hypertension. Circ Heart Fail. 2012;5(1):97-105. 